$10-2009$

\title{
Compromising Anonymous Communication Systems Using Blind Source Separation
}

\author{
Ye Zhu \\ Cleveland State University, y.zhu61@csuohio.edu \\ Riccardo Bettati \\ Texas A \& M University - College Station, bettati@cs.tamu.edu
}

Follow this and additional works at: https://engagedscholarship.csuohio.edu/enece_facpub

Part of the Systems and Communications Commons

How does access to this work benefit you? Let us know!

\section{Publisher's Statement}

(C) ACM, 2009. This is the author's version of the work. It is posted here by permission of ACM for your personal use. Not for redistribution. The definitive version was published in ACM Transactions on Information and System Security, $\{13,1$, October 1, 2009\} http://doi.acm.org/ $10.1145 / 1609956.1609964$

\section{Original Citation}

Y. Zhu and R. Bettati, "Compromising anonymous communication systems using blind source separation," ACM Transactions on Information and System Security (TISSEC), vol. 13, pp. 1-31, 2009.

\section{Repository Citation}

Zhu, Ye and Bettati, Riccardo, "Compromising Anonymous Communication Systems Using Blind Source Separation" (2009). Electrical Engineering \& Computer Science Faculty Publications. 40.

https://engagedscholarship.csuohio.edu/enece_facpub/40

This Article is brought to you for free and open access by the Electrical Engineering \& Computer Science Department at EngagedScholarship@CSU. It has been accepted for inclusion in Electrical Engineering \& Computer Science Faculty Publications by an authorized administrator of EngagedScholarship@CSU. For more information, please contact library.es@csuohio.edu. 


\title{
Compromising Anonymous Communication Systems Using Blind Source Separation
}

\author{
YE ZHU \\ Cleveland State University \\ and \\ RICCARDO BETTATI \\ Texas A\&M University
}

We propose a class of anonymity attacks to both wired and wireless anonymity networks. These attacks are based on the blind source separation algorithms widely used to recover individual signals from mixtures of signals in statistical signal processing. Since the philosophy behind the design of current anonymity networks is to mix traffic or to hide in crowds, the proposed anonymity attacks are very effective.

The flow separation attack proposed for wired anonymity networks can separate the traffic in a mix network. Our experiments show that this attack is effective and scalable. By combining the flow separation method with frequency spectrum matching, a passive attacker can derive the traffic map of the mix network. We use a nontrivial network to show that the combined attack works.

The proposed anonymity attacks for wireless networks can identify nodes in fully anonymized wireless networks using collections of very simple sensors. Based on a time series of counts of anonymous packets provided by the sensors, we estimate the number of nodes with the use of principal component analysis. We then proceed to separate the collected packet data into traffic flows that, with help of the spatial diversity in the available sensors, can be used to estimate the location of the wireless nodes. Our simulation experiments indicate that the estimators show high accuracy and high confidence for anonymized TCP traffic. Additional experiments indicate that the estimators perform very well in anonymous wireless networks that use traffic padding.

Categories and Subject Descriptors: C.2.0 [Computer-Communication Networks]: GeneralSecurity and protection; C.2.2 [Computer-Communication Networks]: Network ProtocolsApplications; K.4.1 [Computers and Society]: Public Policy Issues-Privacy

General Terms: Security, Algorithms

Additional Key Words and Phrases: Anonymous communication, location privacy, blind source separation

This work is partially supported by faculty research development grant (FRD) from Cleveland State University.

Authors' addresses: Y. Zhu, Department of Electrical and Computer Engineering, Cleveland State University, 2121 Euclid Ave, SH 433, Cleveland, OH 44115; email: y.zhu61@csuohio.edu; R. Bettati, Department of Computer Science and Engineering, Texas A\&M University, College Station, TX 77843; email: bettati@cs.tamu.edu. 


\section{INTRODUCTION}

One of the key premises in anonymity systems has been that large crowds protect the anonymity of individuals. Anonymity measures rely on users being able to "hide in a crowd," and the larger the crowd, the easier it is for the individual to hide. This tenet has been underlying early formulations of anonymity metrics (such as the anonymity set size in Kesdogan et al. [1998]) and implicitly most newer metrics as well, such as Serjantov and Danezis [2002] and Diaz et al. [2002]. Empirical results support this assumption, given that larger numbers of participants naturally decrease the signal-to-noise ratio of the footprint of any individual.

In this article we propose data preconditioning methods that allow to partition the set of participants and, therefore, significantly increase the footprint of individuals. We formulate these pre-conditioning methods as a class of attacks, and we apply them to on both wired and wireless anonymity networks. We use blind source separation (BSS), a methodology from statistical signal processing to recover unobserved "source" signals from a set of observed mixtures of the signals. These attacks can be launched against wired anonymous communication networks to unmix traffic and thus affect traditional anonymity such as sender anonymity, receiver anonymity or sender/receiver anonymity. These attacks can also be launched against wireless anonymous communication networks to isolate and identify participants and to compromise location privacy.

\subsection{Flow Separation Attack on Wired Anonymous Communication Networks}

In this article, we discuss flow separation, which aims at separating (as opposed to identifying) flows inside a network, based on aggregate traffic information only. Once flows have been separated into single flows or small groups of flows, subsequent frequency spectrum matching Zhu et al. [2004] or time domain cross-correlation [Levine et al. 2004] can then easily determine additional information about flows, such as the path taken, the ingress and egress points into and out of the network.

Once flows have been separated, information about the traffic content of the flows can be inferred as well. As a practical example, Dusi et al. [2008] describe the difficulty of classifying traffic over SSH tunnels when multiple flows are multiplexed over the same tunnel. Later in this article, we will illustrate how flow separation yields representations of flows that highly correlate to the existing flows. This allows the classification mechanisms described by Dusi et al. [2008] to be applied effectively on aggregates of tunneled flows.

We empirically to show that flow separation attacks are effective for both single mixes and mix networks. We also show that further attacks can make use of the information about the separated flows and so be very effective in reducing anonymity. We analyze the effect of multicast/broadcast traffic on the 
flow separation attack. In contrast to intuition, our analysis and experiments show that the presence of multicast/broadcast traffic significantly helps the attacker to more precisely separate the flows.

\subsection{Location Privacy Attacks on Wireless Anonymous Communication Networks}

With the increasing popularity of 802.11-style wireless networks (WLANs), both in infrastructure and in ad hoc mode, location privacy issues in such networks and in ubiquitous computing environments in general have received great attention. Much recent work has focused on the identification of location privacy risks associated with the use of WLANs and on the implications of weak location privacy (e.g., Cuellar et al. [2004]). Locating a node in a wireless network typically requires first to identify the node (where some identifier is associated with the node, without necessarily disclosing the node's user identity) before proceeding to the proper geographic location. In densely populated networks, node location is difficult without prior identification, since it is impossible to properly attributed traffic to nodes and so to keep the nodes apart. In sparsely populated networks, the identification step is trivial and can be skipped altogether.

Appropriate preconditioning of collected traffic data using flow separation allows an attacker to compromise the location privacy in a densely populated, perfectly anonymized wireless network. The traffic data could be collected by very simple sensors, which only need to monitor packets at MAC level or above, do not require directional capabilities, do not need to distinguish packets or relate network packets to senders or receivers, only require coarse time synchronization support, and require only low-bandwidth links for intersensor communication. (We do not need support for signal-strength measurement on the sensors either.) Such collections of sensors could be realized by a number of WLAN users that collude and exchange information, or by a separate infrastructure of sensor nodes, such as a sensor network. Given these limited required capabilities, we use the sensors to count packets over intervals of given length and to forward the resulting time series of packet counts for analysis to some central location. No information is available about how many nodes are present and sending in the area, and the anonymity measures in the WLAN prevent the sensors from distinguishing packets sent from different nodes.

Our experimental evaluations using a widely accepted packet-level network simulator (ns-2) indicate that (i) the proposed algorithms estimate the node density with high accuracy and that (ii) they estimate node locations with both high accuracy and high confidence. The majority of experiments is performed with the intent to simulate naturally occurring (i.e., TCP) traffic over typical anonymizing wireless networks, such as ANODR [Kong and Hong 2003]. In order to show the effectiveness of the approach, we also simulate a network that uses constant-rate padding of traffic on all nodes. Our experiments indicate that traffic padding is largely useless in this setting: It has no impact on the effectiveness of our estimators for both node density and node location.

We consider these results significant, since they indicate that it is impossible to maintain location privacy in 802.11-style networks against colluding WLAN 
users or networks of sensors that use simple off-the-shelf technology. Our experiments show that crowds are unable to hide individuals in WLAN settings. BSS algorithms can easily and effectively separate packets from different senders, based on packet-count time series only.

\subsection{Statistical Methods for Flow Separation}

The preconditioning methods described in this article rely on BSS [Jutten and Herault 1991], which was originally developed to solve variations of the cocktail party problem, where the goal is to extract one speaker's voice signal given a mixtures of voices, presumably at a cocktail party. BSS algorithms solve the problem by taking advantage of the independence between voices from different speakers. We will show how BSS can be used in wired networks to "unmix" traffic flows Similarly, in wireless networks, we can use BSS algorithms to separate traffic from different wireless nodes. The separated traffic is not in a form that can be directly associated with any sender node. However, we take advantage of spatial diversity in the collected data to reconstruct the path of a flow through a mix network, or the the sender location in a wireless network, based on the separated traffic.

While the number of "mixed flows" through a wired mix are known (assuming one knows the number of ports of the mix router) the number of senders in a wireless network is typically unknown. In Zhu and Bettati [2007], we describe how we use principal component analysis (PCA) to estimate the number of sending nodes.

\subsection{Structure of the Article}

The remainder of this article is organized as follows: Section 2 reviews the related work. In Section 3, we introduce the BSS algorithms. In Section 4, we introduce the flow separation attack for the wired anonymity networks. Section 5 describes the attacks to compromise location privacy in wireless anonymity networks. In Sections 6 and 7, we use ns-2 simulation experiments to show the effectiveness of the flow separation attack. We evaluate the flow separation attack against a nontrivial mix network in Section 8. Section 9 evaluates the performance of location privacy attacks for wireless anonymity networks. Section 10 discusses countermeasures for flow separation attack and further attacks on location privacy. We conclude this article in Section 11. An extended version of this article, with additional information about experimental results, is available in form of a companion technical report [Zhu and Bettati 2007].

\section{RELATED WORK}

\subsection{Traditional Wired Anonymity Networks and Anonymity Attacks}

Reviving Chaum's idea to use mixes to reroute messages, Helsingius [1996] implemented the first Internet anonymous remailer, which is a single-application proxy and replaces the original email's source address with the remailer's address. Hughes and Finney [Parekh 1996] built the cypherpunk remailer, a distributed mix network with reply functions and encryption. Gülcü and Tsudik 
[1996] developed a relatively complete anonymous email system, called Babel. Mixminion's design [Danezis et al. 2003] considers a large set of attacks that researchers have found [Serjantov et al. 2002; Berthold and Langos 2002; Berthold et al. 2000; Raymond 2001].

Low-latency anonymity systems have been developed for flow-based traffic in the Internet. A typical example is Tor [Dingledine et al. 2004], the secondgeneration onion router, developed for circuit-based low-latency anonymous communication.

Recently, ad hoc anonymizing networks have been discovered to hide the communication to command-and-control nodes in botnets: The Storm [Porras et al. 2007] botnet, for example, is assumed to use multiple layers of Nginx proxies to hide the command-and-control infrastructure from view. An additional layer of Nginx nodes then act as reverse proxies and uses fast-flux to hide the location of the command server despite using hard URLs for the bot nodes when "calling home" to the controller. The Storm command-and-control system is an example of effective server hiding, made possible by redirection mechanisms in combination with jurisdictional boundaries. As botnets evolve, it is to be expected that they will develop more sophisticated anonymization protocols, which will take advantage of the very large number of bot nodes available.

Exploiting the premise that communication patterns are difficult to efficiently hide, a large amount of work has addressed the effectiveness of messagebased anonymity attacks [Serjantov et al. 2002], a class of flow-based anonymity attacks have been proposed in the literature. Examples are intersection attacks [Danezis and Serjantov 2004], timing attacks [Levine et al. 2004], Danezis's attack on continuous mixes [Danezis 2004], and the flow correlation attack [Zhu et al. 2004]. The timing attack [Levine et al. 2004] uses time domain crosscorrelation to match flows given the packet timestamps of the flow. Danezis's attack on the continuous mix [Danezis 2004] takes advantage of the fact that packets are independently delayed at the mix, and the effect of the mix can be modeled as a convolution on the packet times. The flow correlation attack [Zhu et al. 2004] employs statistical methods to detect TCP flows in aggregate traffic. The flow correlation attack can achieve high detection rates for all the mixes described in Serjantov et al. [2002] and for continuous mixes. The flow separation attack proposed in this article belongs to the flow-based anonymity attacks.

\subsection{Wireless Anonymity Networks and Location Privacy}

Following the realization that serious privacy issues are at stake when location information is accessible in many pervasive applications and wireless networks (see, e.g., the work of the IETF Working Group on Geographic Location/Privacy (geopriv) [Cuellar et al. 2004]), several communication systems to preserve anonymity and location privacy in ad hoc and infrastructure-based wireless networks have been proposed. ANODR [Kong and Hong 2003] protects route anonymity by an onion-based encryption and routing protocol. The data transmission in ANODR is based on broadcast, and identity disclosure is prevented by the use of broadcast MAC addresses. MASK [Zhang et al. 2005] also provides 
a solution for anonymous routing in wireless ad hoc network. In particular, forging packets and inserting dummy packets are proposed to counter traffic analysis. For infrastructure-based networks, Gruteser et al. [2003] proposed the use of disposable MAC addresses in order to prevent tracking of mobile hosts.

Numerous articles have been published on locating wireless nodes. Many of them are based on the characteristic of physical signals, such as Received Signal Strength (RSS) [Bahl and Padmanabhan 2000], Angle of Arrival (AOA) [Niculescu and Nath 2004], and time of arrival (TOA) [Guvenc et al. 2003]. Complex processing methods on collected data are needed to deal with the physical signal's nonlinearity, noise, and the complex correlations caused by multipath effects, interference, and absorption. Elnahrawy et al. [2004] point out a number of fundamental limits associated with the use of signal strength and claim that these limits are unlikely to be transcended.

Senders can easily counter location estimation attacks based on signal strength by modulating the transmission power. This has been proposed in Whisper [Cai et al. 2005]. Location privacy attacks using AOA data assume that sensors have directional capabilities, which adds greatly to the cost of the sensor network. One objective of this article is to illustrate how appropriate preconditioning of the collected traffic data renders most of current anonymity methods for wireless networks, such as encryption, MAC address hiding, signal power fluctuations, link padding, and others are ineffective in 802.11-style setting. For this, we show that we need not make use of information that can be hidden by anonymity measures, such as header data, sender or receiver information, packet data, signal strength, or directional information.

In general, schemes that rely on physical-level, analog signals require large volumes of data to be transfered over the wireless sensor network for further analysis. In comparison, the schemes proposed in this article rely on highly aggregated packet-count data which can be easily propagated across a lowbandwidth infrastructure. Spatial and temporal redundancy of the packetcount data from different sensors can be exploited to further reduce traffic volume by using appropriate compression methods.

\section{BLIND SOURCE SEPARATION}

BSS is a methodology in statistical signal processing to recover unobserved "source" signals from a set of observed mixtures of the signals. The separation is called "blind" to emphasize that the source signals are not observed and that the mixture is a black box to the observer. While no knowledge is available about the mixture, in many cases, it can be safely assumed that source signals are independent. A very nice introduction to the statistical principles behind BSS is given in Cardoso [1998]: In its simplest form, the BSS model assumes $n$ independent signals $F_{1}(t), \ldots, F_{n}(t)$ and $n$ observations of mixture $O_{1}(t), \ldots, O_{n}(t)$ where $O_{i}(t)=\sum_{j=1}^{n} a_{i j} F_{j}(t)$. The goal of BSS is to reconstruct the source signals $F_{j}(t)$, using only the observed data $O_{i}(t)$, the assumption of independence among the signals $F_{j}(t)$. Given the observations $O_{i}(t)$, BSS techniques estimate the signals $F_{j}(t)$ by maximizing the independence between the estimated 
signals. The common methods employed in BSS are minimization of mutual information [He et al. 2000], maximization of nongaussianity [Hyvarinen and Oja 1997] and maximization of likelihood [Pham et al. 1992].

In our experiments, we use a powerful BSS algorithm proposed in Cruces and Cichocki [2003]. This algorithm can jointly optimize several statistics of the same order, and it combines advantages of other effective techniques, such as Fast-ICA [Hyvarinen and Oja 1997] and SOBI [Belouchrani et al. 1997]. The algorithm has been shown to perform well when the amount of data available is small.

\section{FLOW SEPARATION IN MIX NETWORKS}

\subsection{Network Model and Threat Model}

Mix and Mix Network. A mix is a relay device for anonymous communication. A single-mix network can achieve some level of communication anonymity: The sender of a message attaches the receiver address to a packet and encrypts it using the mix's public key. Upon receiving a packet, the mix decrypts the packet using its private key. Different from an ordinary router, a mix usually will not relay the received packet immediately. Rather, it will attempt to perturb the flows through the mix in order to foil an attacker's effort to link incoming and outgoing packets or flows. It does this, typically, in three ways: First, it reencrypts the packet to foil attacks that attempt to match packets in the payload data domain. Then, it reroutes the packet to foil correlation attacks that rely on route traceback. Finally, it perturbs the flows in the time domain through batching, reordering, and link padding. Batching collects several packets and then sends them out in a batch. The order of packets may be altered as well. Both these batching techniques are important in order to prevent timing-based attacks. Different batching and reordering strategies are summarized in Serjantov et al. [2002] and Zhu et al. [2004].

Most low-latency anonymity systems do not employ batching, reordering, and padding strategies. For example, Onion Router [Goldschlag et al. 1999], Crowds [Reiter and Rubin 1998], Morphmix [Rennhard and Plattner 2002], P5 [Sherwood et al. 2002], and Tor [Dingledine et al. 2004] do not use any batching and reordering techniques.

A network may consist of multiple mixes that are interconnected by a network such as the Internet. A mix network may provide enhanced anonymity, as payload packets may go through several mixes so that if one mix is compromised, anonymity can still be maintained. In the following, we will use the term mix to mean either single-node mixes, or mix cascades, or networks of mixes.

Threat Model. We assume a passive adversary whose capabilities are summarized as follows.

(1) The adversary passively observes a number of input and output links of a mix or among a collection of mixes, collects the packet arrival and departure times, and analyzes them. This type of attack can be easily staged 
on wired and wireless links [Howard 1998] by a variety of agents, such as governments or malicious ISPs.

(2) For simplicity of discussion, we assume a partially global adversary, that is, an adversary that has observation points on a subset of links between mixes in the mix network. While this assumption seems overly strong, it is not. An attacker with access to only a small number of points will naturally aggregate mixes for which it has no observation points into super-mixes.

(3) The adversary cannot correlate (based on packet timing, content, or size) an individual packet on an input link to another packet on an output link based on content and packet size. This is prevented by encryption and packet padding, respectively.

(4) We focus on mixes that operate as simple proxies. In other words, no batching or reordering is used. Link padding (with dummy packets) is not used either. This follows the practice of some existing mix networks, such as Tor [Dingledine et al. 2004], and other obfuscation mechanisms, such as SSH tunnels and the cascaded Nginx proxies in the Storm botnet.

(5) Finally, the adversary aims to recover the a priori unknown individual flows from the aggregate of indistinguishable traffic. Once a flow or a number of flows have been recovered, the attacker can proceed to either classify the content of the flow, or to compute the path of the flows, or the end-to-end communication matrix, or other measures of interest.

\subsection{Flow Separation}

In this section, we will first define the problem in the context of BSS and then describe how to apply the flow separation method in a wired mix network.

4.2.1 Flow Separation as a BSS Problem. We define a flow to be a series of packets that are exchanged between a pair of hosts. Such a flow can be a single TCP or UDP connection, or can be carried by (striped over) several connections, both UDP or TCP. In this article, we limit ourselves to flows whose packets all flow along a single path. In other words, while packets from different flows can take different paths, all the packets from a single flow follow the same path. We define an aggregate flow at the link-level to be the sum of the packets (belonging to different flows) on the link. We define the aggregate flow at mix-level to be the sum of packets through the same input and output port of a mix. Unless specified otherwise, in the remaining of this article, the word "flow" means "mix-level aggregate flow" for brevity.

In this article, we will show that for the attacker who tries to break the anonymity of a mix, it is very helpful to separate the flows through the mix based on the observation of the traffic. The separation of the flows through the mix can recover the traffic pattern of individual flows or small groups thereof. This fine-granularity information can be used to increase the effectiveness of further analysis, such as the frequency spectrum matching attack described in Section 4.2.2 or the time domain cross-correlation attack [Levine et al. 2004] for flow reconstruction, or application characterization over encrypted tunnels [Dusi et al. 2008], or other flow confidentiality situations. 


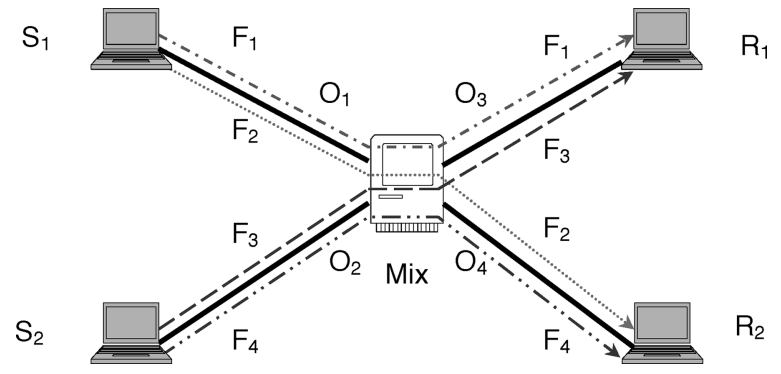

Fig. 1. An example for Flow Model.

In this article, we are interested in the traffic pattern carried in the time series of packet counts during a sequence of identical-length intervals of length $T$. For example, in Figure 1, the attacker can get a time series $O_{1}=\left[o_{1}^{1}, o_{2}^{1}, \ldots, o_{n}^{1}\right]$ of packet counts by observing the link between Sender $S_{1}$ and the mix. We use $n$ to denote the sample size in this article. The attacker's objective is to recover the packet count time series $F_{i}=\left[f_{1}^{i}, f_{2}^{i}, \ldots, f_{n}^{i}\right]$ for each flow. For the simplest case, we assume that (i) there is no congestion in mix and that (ii) the time series can be synchronized. (We will relax both assumptions in later sections.) In the example of Figure 1, the time series $F_{1}$ is contained in both time series $O_{1}$ and $O_{3}$ that is, $O_{1}=F_{1}+F_{2}, O_{3}=F_{1}+F_{3}$. In general, for a mix with $j$ input ports, $k$ output ports, and $m$ mix-level aggregate flows, we can rewrite the problem in vector-matrix notation,

$$
\left[O_{1}, O_{2}, \ldots, O_{j+k}\right]^{T}=\mathbf{A}_{(j+k) \times m}\left[F_{1}, F_{2}, \cdots F_{m}\right]^{T}
$$

where $\mathbf{A}_{(\mathbf{j}+\mathbf{k}) \times \mathbf{m}}$ is called mixing matrix in the BSS problem. Since the aggregate flows through the mix are independent from each other-they come from different sources-we can use any of the methods mentioned in Section 3 to solve the problem. Even the flows from a single host, such as $F_{1}$ and $F_{2}$, can be regarded as independent as they follow different paths and are controlled by different sockets. This independence assumption is of course only valid as long as Sender $S_{1}$ is not heavily overloaded, since otherwise one flow would influence the other.

In the following, we need to keep in mind that flow separation often is not able to separate individual flows. Rather, mix-level aggregates flows that share the links at the observation points form what we call the minimum separable unit.

Basic BSS algorithms require the number of observations to be larger than or equal to the number of independent components. For flow separation, this means that $j+k \geq m$, where $j$ and $k$ denote the number of observations at the input and output of the mix, respectively, and $m$ denotes the number of flows. Advanced BSS algorithms [Hyvarinen and Inki 2002] target overcomplete bases problems and can be used for the case where $m>j+k$. But they usually require that $m$, the number of independent flows, be known. Since all the mix traffic is encrypted, and all packets padded to the same length, it is hard for the attacker to estimate $m$. We assume that $m=j+k$. The cost of the assumption is that 
some independent flows can not be separated, that is, they remain mixed. We will see that this is not a severe constraint, in particular not in mix networks where flows that remain mixed in some separations can be separated using separation results in neighboring mixes.

Unless there is multicast or broadcast traffic through the mix, the $j+k$ observations will have some redundancy, because the summation of all the observations on the input ports are equal to the summation of all the observations on the output ports. In other words, the row vectors of the mixing matrix are linearly dependent. Again, the cost of the redundancy is that some independent flows are not separated.

The flow estimation generated by BSS algorithms is usually a scaled version of the actual flow (of its time series, actually). Sometimes, the estimated flow may be of different sign than the actual flow. Scaling does not affect the frequency components of the time series, so frequency matching can be used to further analyze the generated data.

Furthermore, since the elements of the estimated mixing matrix are not binary, it is not straightforward to tell the direction of each aggregate flow. Some heuristic approach can be used, but we leave this to further research.

When monitoring points can be placed at some locations inside the network, separation results at different locations can be compared, and common separated components can be identified if such components exist. This can be used to reconstruct the path of flows or to simply strengthen the confidence in the results of the flow separation step.

4.2.2 Frequency Matching Across Neighboring Mixes. The result of flow separation is a set of mix-level aggregate flows that have been determined to traverse the mix. Each separated aggregate flow is identified by a time series of packet counts. As pointed out earlier, some of these aggregate flows may represent multiple actual flows that could not be separated. Aggregate flows can be further separated by comparing BSS results across multiple mixes.

Frequency spectrum matching has shown to be particularly effective to further analyze the traffic. The rationale for the use of frequency matching is fourfold: First, the dynamics of a flow, especially a TCP flow [Huang et al. 2001], is characterized by its periodicities. By matching the frequency spectrum of a known flow with the frequency spectrums of estimated flows obtained by BSS techniques, we can identify the known flow with high accuracy. Second, frequency matching removes the zero-frequency component, and so easily eliminates ambiguities introduced by the scaling in the estimated time series. Third, frequency spectrum matching can also be applied on the mix-level aggregate flows, since the different frequency components in each individual flows can characterize the aggregate flow. Fourth, the low-frequency components of traffic are often not affected by congestion as they traverse multiple switches and mixes. This is particularly the case for TCP traffic, where the frequency components are largely defined by the behavior at the end hosts. In summary, frequency spectrum analysis has excellent prerequisites to be highly effective. 
Even if no information is available about individual flows, the attacker can easily determine if there is communication between two neighboring mixes. Matching the estimated aggregate flows across neighboring mixes can give attackers more information, such as how many aggregate flows are traversing the next mix. In a mix network, an aggregate flow through a mix may split into aggregate flows of smaller size, multiplex with other aggregate flows, or both. By matching the estimated aggregate flows on mixes along estimated paths, and by comparing the results against those of other mixes, the attacker can detect such splitting and multiplexing. Based on the information gathered, and if sufficient monitoring points are available in the network, the attacker can eventually get a detailed map of traffic in a mix network, without having access to information about any individual flow. In Section 8, we show a traffic map of

flows in a large network, which was obtained from BSS followed by aggregate flow matching.

\section{COMPROMISE LOCATION PRIVACY IN WIRELESS ANONYMITY NETWORKS}

Wireless anonymity networks naturally offer themselves very well to a BSS analysis. In a wireless setting with passive sensors, the packet flows originating at the senders give rise to the unobserved signals, and the packets overheard by the sensors produce the observed signals. Finally, the wireless medium acts as mix matrix. BSS can, therefore, be used to separate senders and to study how this supports follow-up attacks, such as localization of senders.

\subsection{Network Model and Threat Model}

In the following, we assume a passive attacker who is interested in determining the number of nodes in the network (node density) and the geographic location of the nodes (node location). Disclosure of node location may aid privacy attacks, but may be used in other settings as well. For example, low-cost intrusion detection schemes for ad hoc networks can perform node density estimations at deployment time and determine if active intruders are present. If so, location estimations can support the localization of the intruder.

Network Model. We assume a set of wireless nodes (simply called nodes in the following) that communicate over an ad hoc WLAN using an 802.11-style MAC protocol. We assume that all communications are perfectly anonymized. For example, all communications are broadcast-based so that the anonymity attacker cannot identify the source and destination of a MAC frame [Kong and Hong 2003; Zhu et al. 2004]. Similarly, MAC addresses can be recycled [Gruteser and Grunwald 2003] to achieve the same effect. We also assume that all the packets inside the wireless network are encrypted.

Threat Model. As a result, no information is divulged to external observers either through packet data or header content. In addition, we assume that nodes are able to manipulate signal power [Cai et al. 2005] so as to render any observed signal strength information difficult to use. We derive the thread model from that described for the wired case in Section 4.1 and adapt it to the 


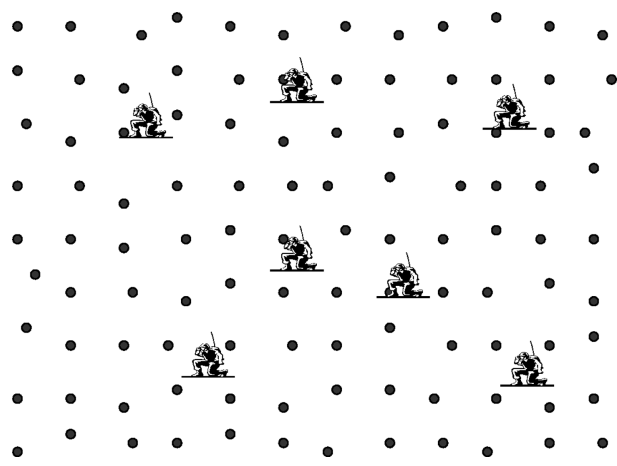

Fig. 2. Threat Model.

case of anonymized wireless systems, similar to Kong et al. [2003]. We assume that the communication between nodes is observed by a network of low-cost sensors scattered around a field. The sensors can be either WLAN receivers of a set of colluding users in the area, or they can be deployed as a separate sensor network infrastructure. The attacker collects packet timing information from the sensors in the field for analysis. We summarize the capabilities of the passive attacker as follows.

(1) Sensor nodes have off-the-shelf 802.11 receivers.

(2) No signal strength information is available.

(3) No directional information is available.

(4) Time synchronization across sensor nodes is insufficient to allow for signalpropagation-based location estimation.

(5) A sensor cannot associate a packet with a sender or receiver node.

(6) The location of each sensor is known.

(7) The sensor are scattered randomly, but uniformly, over the networked area of interest, as shown in Figure 2.

(8) The communication between sensors does not interfere with the communication between wireless nodes.

\subsection{Data Collection and Preprocessing}

The data collected from each sensor is a time series of counts of the packets "overheard" by the sensor. (While sensors cannot decrypt the packets or even associate packets with a mobile node, they can mark the time when a packet is received, and so count the number of packets received over any interval.) We use the time series $S_{i}=\left[s_{i}^{1}, s_{i}^{2}, \ldots, s_{i}^{l}\right]$ to denote the series of packet counts detected by Sensor $i$ during a sequence of intervals of length $T$ each. Since there may be several wireless nodes in the field, the packet counts on each sensor may contain packets from several wireless nodes. Similarly, the same packet may be counted by multiple sensors.

As for any data-gathering application on sensor networks, power consumption and bandwidth limitations are important design issues. Only packet counts 
are collected from the sensor, and so the resulting amount of data is significantly less than from collecting, say the timestamp of each packet. In addition, we can use data compression or coding schemes designed for sensor networks such as MEGA [von Rickenbach and Wattenhofer 2004] to exploit any remaining spatial redundancy across neighboring nodes or temporal redundancy at individual nodes.

\subsection{Node Location Estimation}

In a network where all packets are perfectly anonymized, the estimation of the location of sender nodes has only aggregated packet data available, since packets sent by different nodes cannot be distinguished. In the following, we describe how we use BSS to deaggregate the packet-count time series collected at a group of sensors into an estimation of the per-node packet-count time series $M_{j}$ of sender node $j$. Based on the estimated per-node time series, we then use a proximity-based scheme to estimate the location of nodes.

5.3.1 BSS. While the goal of BSS in this context is to re-construct the original signals $M_{i}$ (the time series of packet counts sent by individual nodes), in practice the separated signals (we call these components) are sometimes only loosely related to the original signals. We categorize these separated components into three types. In the first case, the component is correlated to some signal $M_{i}$. We call this type of component individual component. In the second case a component may be correlated to an aggregate of signals from several nodes. This happens when the packets of more than two wireless nodes can be "heard" by all the sensors. In such a case, the BSS algorithm would not be able to fully separate the signal mixture into the individual components. Rather, while some components can be successfully separated, others remain aggregated. In the third case, components may represent noise signals. Noise in our case can be caused by packet collisions that prevent some sensors from "hearing" some packets. Noise can also emerge as artifact from generating the packet timing sequences. For example, a packet may be counted in the $i^{\text {th }}$ interval for some sensor, while for some other sensor, the same packet may be counted in the $(i+1)^{t h}$ interval due to transmission delay or imperfect synchronization. For brevity, we call the second type aggregate component and the third type noise component.

5.3.2 Node Location Estimation Algorithm. An algorithm to estimate the location of sender nodes would consist of three steps. In a first step, we partition the network area into a set of mini areas. In order to maximize both spatial resolution and spatial diversity in the following steps, we should choose the size of the mini areas to be small but sufficiently large so that most subareas contain at least one sensor. We group the nodes in each $c \times c$ neighboring set of mini areas into sensor block, as shown in Figure $3 .{ }^{1}$ Neighboring blocks are overlapping, and as a result, sensors generally belong to several sensor blocks. (For the case

\footnotetext{
${ }^{1}$ In general, mini-areas and sensor blocks can be irregularly shaped. In Figure 3, we draw them to be quadratic.
} 


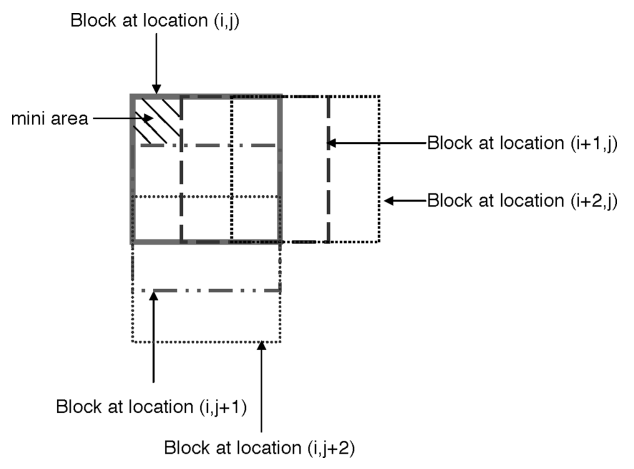

Fig. 3. Sensor blocks.

of a quadratic blocks, most sensors belong to $c^{2}$ blocks.) For each block of sensors, we sequentially apply a BSS algorithm to recover the packet traces of mobile nodes in the sensing range. As a result of this block-by-block separation step, we are left with a large set of components, as described in Section 5.3.1. Many of these components are either aggregates or noise components. In a second step, we eliminate the latter by identifying components that appear in several blocks. This is achieved by identifying clusters of similar components across all blocks. This clustering step generates a set of components that have been detected by several blocks of sensors and are likely to be similar to the original signals.

At this point, the senders have been identified, and the attacker can proceed to extract additional information. In the following, we focus on localization of the senders. In the third and last step, we estimate the location of the senders by intersecting the sensing ranges for all blocks that have separated components that are highly correlated to the original signals.

We describe the three steps (separation, clustering, intersection) in more detail:

Separation Step. For each sensor block, we apply BSS to recover a set of $s$ components, as described in Section 5.3.1 for a sensor block with $s$ sensors. We use $R_{i}^{j}$ to represent the $j^{\text {th }}$ recovered component from the $i^{t h}$ sensor block.

Clustering Step. We eliminate those components that are likely to be noise or aggregate components. For this, we use the following heuristic: If a component represents a real signal, the same component has likely been detected and separated in at least a similar form by more than one sensor block. In contrast, a component that was generated because of some interference or other artifacts has likely been generated by a single block only.

Based on this heuristic, we identify clusters of similar components by using the cross-correlation coefficient as measure for similarity and define the distance between two components as follows:

$$
D\left(R_{i}^{p}, R_{j}^{q}\right)=1-\left\|\operatorname{corr}\left(R_{i}^{p}, R_{j}^{q}\right)\right\|
$$




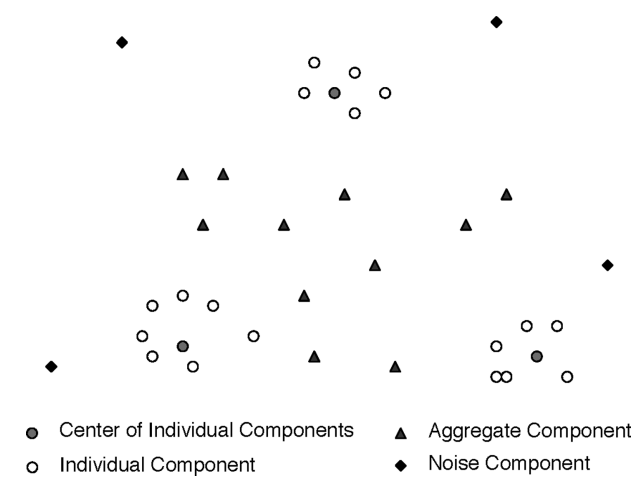

Fig. 4. Visualization of distance between separated components.

where $R_{i}^{p}$ denotes the $p^{\text {th }}$ component recovered from the $i^{\text {th }}$ sensor block $B_{i}$, and $\operatorname{corr}(X, Y)$ denotes the correlation coefficient of components $X$ and $Y$. (We use the absolute value of the cross-correlation because the separated components may be of different sign than the actual time series.) As a result, the highly correlated (similar) components will cluster together. Figure 4 uses a two-dimensional representation to further illustrate the rationale for the clustering approach in this step. As shown in this figure, the individual components form clusters. The aggregate components on the other hand scatter in-between these clusters. The noise components are distant both from each other and from the other components.

We select the center components $R_{1}, \ldots, R_{K}$ of the $K$ largest resulting clusters, where $K$ is the estimated number of nodes in the area. The value for $K$ is either known a priori or is estimated using a highly accurate method based on PCA, which we describe in Zhu and Bettati [2007] in detail. We note that it is highly unlikely that either aggregate or noise components are selected as center components: (i) Aggregate components are unlikely in the center of clusters, and (ii) noise is local to a small group of sensors at best and gives rise to small clusters. As a result, the $K$ selected center components will be highly correlated to the packet count times series $M_{1}, \ldots, M_{K}$ of the nodes in the network.

Intersection Step. We locate a sending node by intersecting the sensing ranges of blocks that are likely to "hear" the node. For this, we select sensor blocks that have components that are closely correlated with the likely time series of the nodes in the area. The rationale is that for the sensors in a sensor block to hear a node, they must have sensed a signal that is at least similar to the signal generated by the node. This means that sensor blocks with components that correlate with any of the $K$ center components are likely to hear a sending node. Therefore, we determine the likely location of a node by geographically intersecting the sensing areas of the sensors in those sensor blocks that have highly correlated component with a center component determined in the preceding clustering step.

A straightforward correlation of the components gives poor results for two reasons. First, sensor blocks in the immediate neighborhood of a sender node 
often display insufficient spacial diversity to successfully separate the component representing the sender node packet time series; therefore, they must be discarded. Second, simply correlating the components of the sensor block with a particular center component may lead to too many false positives because geographically very distant components may occasionally correlate with a particular center component. For example, correlated components may appear along the the multihop path of a connection in an ad hoc network and give rise to geographically distant sensor blocks that are erroneously classified as being able to hear the node. In such cases, the geographical area intersection approach fails to locate the node. We address these problems by borrowing techniques and terminology from image processing.

For each center component $R_{k}$, we generate a "image matrix" $I M G_{k}$ of correlation coefficients (the "intensity") as follows: Each entry in the matrix $I M G_{k}(i, j)$ represents the maximum correlation between the center component $R_{k}$ and all $c^{2}$ components of the block, say $B_{u}$, at location $(i, j)$ :

$$
I M G_{k}(i, j)=\max _{1 \leq p \leq c^{2}}\left(\operatorname{corr}\left(R_{u}^{p}, R_{k}\right)\right.
$$

We then apply edge detection to partition the matrix into geographically contiguous regions, each with components that correlate with the center component $R_{k}$. We discard the regions that are not sufficiently correlated with the center component. (A detailed discussion of the threshold selection in this step is given in Zhu and Bettati [2007].)

We intersect the sensing area of sensor blocks in the remaining regions as follows: Sort the points $I M G_{k}(i, j)$ in the remaining regions in order of decreasing intensity. Starting with the highest-intensity point, add subsequent points by intersecting their sensing range. Stop when you either run out of points or the new point's sensing area is disjoint from the computed intersection area, causing the new intersection area to disappear. The resulting intersection area is the suspected area of location of a node.

\section{EVALUATION OF FLOW SEPARATION ATTACK ON A SINGLE MIX WITH DIFFERENT COMBINATIONS OF TRAFFIC}

Whenever we have access to monitors at the boundary of some network, we treat the network as a single mix (which we call super-mix), independently of whether the network contains one or more mixes. Whenever we can plant additional monitors inside the network, we are de facto partitioning the single-node mix view into that of a mix network. In this section, we evaluate the single-mix case, and we will elaborate the results on the network case in Section 8.

\subsection{Experiment Set-up}

Figure 5 shows the experimental network set-up with a single mix. We use ns-2 to simulate the network. The links in the figure are all of $10 \mathrm{Mbit} / \mathrm{s}$ bandwidth and $10 \mathrm{~ms}$ delay $^{2}$ if not specified otherwise. The mix under study has two input

\footnotetext{
${ }^{2}$ Senders and receivers can be at a large distance from the mix, potentially connecting through several routers and switches.
} 


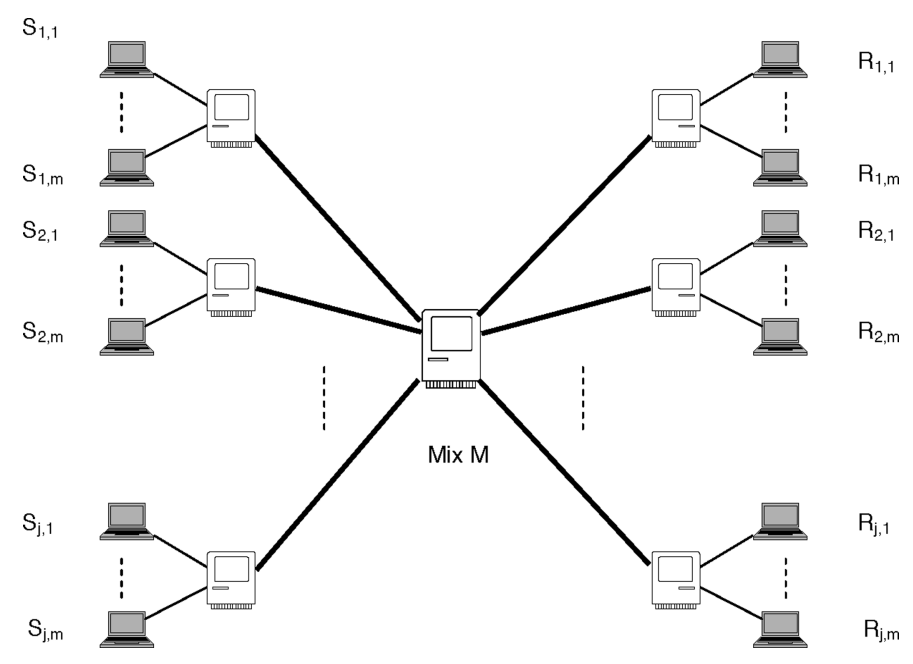

Fig. 5. Experiment set-up for single mix.

ports and two output ports and four aggregate flows passing through the mix, as shown in Figure 1. We study mixes with more than two ports in Section 7. Unless specified otherwise, we use time observation intervals of $32 \mathrm{~s}$ length and sample interval of $10 \mathrm{~ms}$ length, resulting in time series of size $n=3,200$. Similar results were obtained for shorter observations as well.

\subsection{Metrics}

In the following, we will use two metrics to evaluate the accuracy of the flow separation. The metrics compare the separated flows with the actual flows in the time domain and the frequency domain, respectively.

We use mean square error (MSE) to match separated and actual flows in the time domain as follows: Let $F_{A}=\left[f_{1}^{A}, f_{2}^{A}, \ldots, f_{n}^{A}\right]$ represent the time series of the actual flow and $F_{B}=\left[f_{1}^{B}, f_{2}^{B}, \ldots, f_{n}^{B}\right]$ represent the time series estimated by the BSS algorithm. To match the time series $F_{A}$ with $F_{B}$, we first scale and lift $F_{B}$ so that both series have the same mean and variance.

$$
F_{B}^{\prime}=\frac{\operatorname{std}\left(F_{A}\right)}{\operatorname{std}\left(F_{B}\right)} \cdot\left(F_{B}-\operatorname{mean}\left(F_{B}\right) \cdot[1,1, \ldots, 1]\right)+\operatorname{mean}\left(F_{A}\right) \cdot[1,1, \ldots, 1],
$$

where $\operatorname{std}(F)$ and mean $(F)$ denote the standard deviation and average of the time series $F$, respectively. The mean square error is defined as follows:

$$
\varepsilon_{A, B}=\frac{\left\|F_{A}-F_{B}^{\prime}\right\|^{2}}{n} .
$$

Since the times series $F_{B}$ can also be a flipped version of $F_{A}$, we need to match $F_{A}$ with $-F_{B}$ as well.

We use a second metric to evaluate how well the separated Flow $F_{B}$ matches the actual flows in the frequency domain. (In Section 4.2.2, we describe how the correlation of actual and separated flows in the frequency domain is used when 


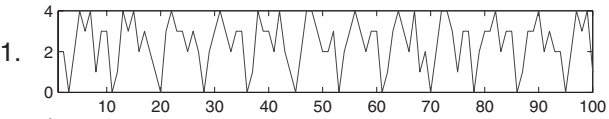

2.

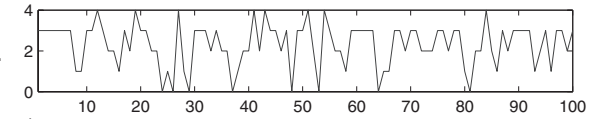

3.

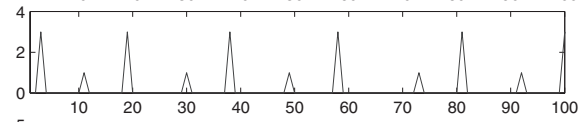

4.

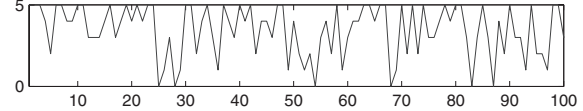

(a) Actual flows

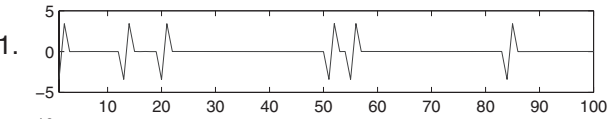

2.

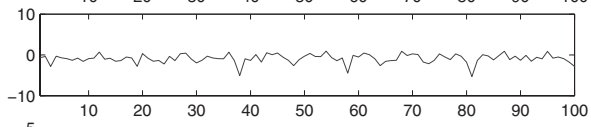

3.

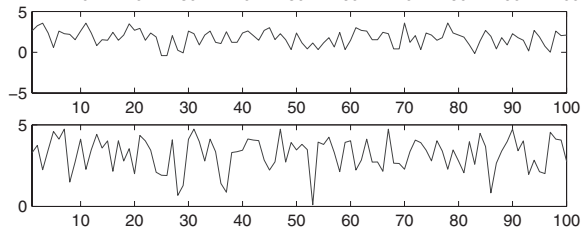

(b) Separated flows

Fig. 6. Example of flow separation for different types of traffic.

we analyze systems with multiple mixes.) To evaluate the performance in frequency domain, we use the second metric called frequency spectrum matching degree. We define the matching degree between $F_{B}$ and $F_{A}$ to be the absolute value of correlation between the spectrum of the separated flow $F_{B}$ and the spectrum of the actual flow $F_{A}$.

\subsection{Different Types of Traffic}

In this experiment, four aggregate flows including one FTP flow, one sequence of HTTP requests, and two on/off UDP flows are passing through the mix. The parameters for the flows are as follows: Flow 1: FTP flow, with round trip time around $80 \mathrm{~ms}$. Flow 2: UDP-1 flow, on/off traffic, with burst rate 2,500kbit/s, average burst time $13 \mathrm{~ms}$ and average idle time $6 \mathrm{~ms}$. Flow 3: HTTP flows, with average page size 2,048 byte. Flow 4: UDP-2, on/off traffic with burst rate $4,000 \mathrm{kbit} / \mathrm{s}$, average burst time $12 \mathrm{~ms}$ and average idle time $5 \mathrm{~ms}$. All the random parameters for the flows are exponentially distributed. The flows traverse the mix as shown in Figure 1.

Figure 6 shows portions of the actual times series (Figure 6(a)) and of the separated time series (Figure 6(b)). From the figures, it is apparent that the flipped version of the actual Flow 3 (HTTP flows) is contained in the separated Flow 2. We also observe the resemblance between actual Flow 1 (FTP flow) and separated Flow 4. Separated Flow 1 is clearly not close to any actual flows. This is caused by the redundancy contained in the observations, as described in Section 4.2.1.

Figure 7 shows the separation accuracy using the two metrics defined earlier. We note in Figure 7(b) that both the separated flow and its flipped time series is compared against the actual flows. Both metrics can identify the FTP flow, the HTTP flows, and Flow UDP-2. But the two metrics disagree on Flow UDP-1: while Flow UDP-1 correlates well in the frequency domain with separated Flow 3 (indicated by the highest frequency spectrum matching degree for Flow UDP-1 in Figure 7(a)) in the time domain it best matches with separated Flow 4(flipped) (indicated by the lowest MSE for Flow UDP-1 in Figure 7(a)). 


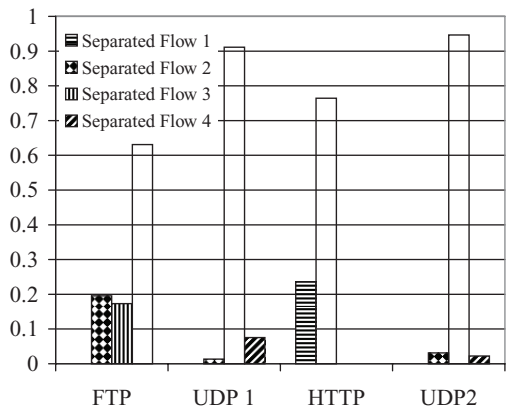

(a) Frequency spectrum matching degree

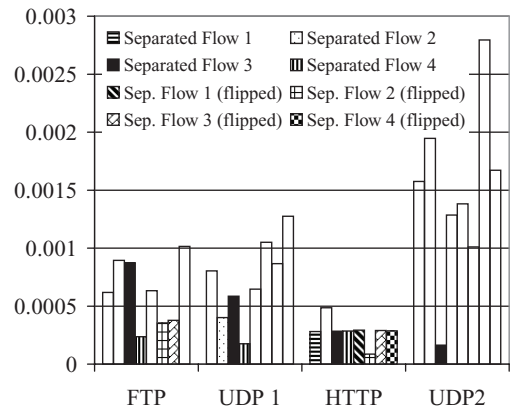

(b)MSE

Fig. 7. Performance of flow separation for different types of traffic.

1.

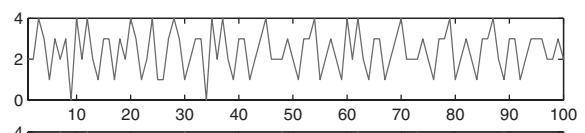

2.

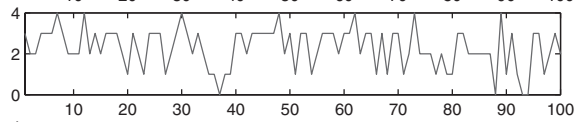

3.

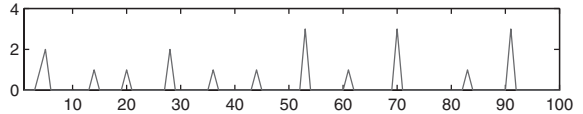

4

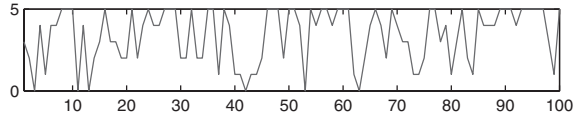

(a) Actual flows
1.

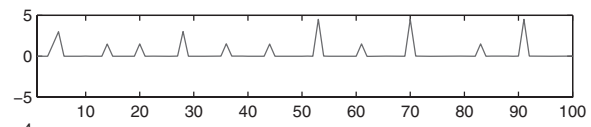

2.

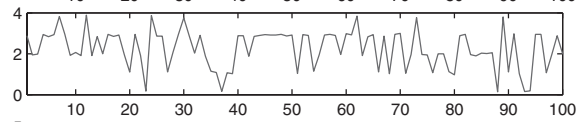

3.

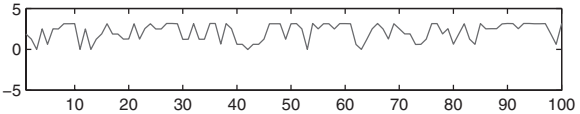

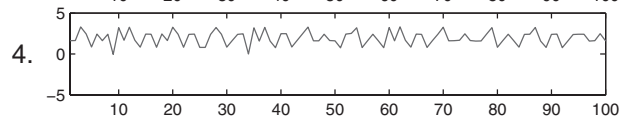

(b) Separated flows

Fig. 8. Example of flow separation for different types of traffic (with multicast traffic).

This is because of the redundancy in the observations, and the two UDP flows can not be separated. MSE fails for this case, since it is designed for one-toone flow matching, while frequency spectrum matching is more suitable for matching of flows against aggregates. The latter case is more common in the context of flow separation, where often aggregates of flows cannot be completely separated.

\subsection{Different Types of Traffic with Multicast Flow}

In this experiment, the Flow UDP-1 in the previous experiment is multicast to both output ports.

Portions of the actual flows and the estimated flows are shown in Figure 8. We observe the correspondence between the actual flows and estimated flows easily. In comparison with the previous experiment, we conclude that multicast flows can help the flow separation, as they eliminate redundant observations.

The MSE performance results in Figure 9 show that the flows are successfully identified. Frequency spectrum matching successfully determine the FTP and HTTP flows but does not perform well on the UDP flows. This is because the 


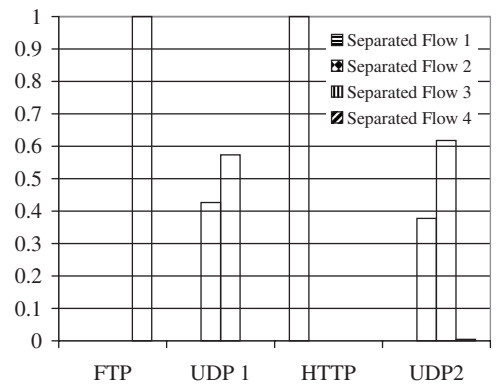

(a) Frequency spectrum matching degree

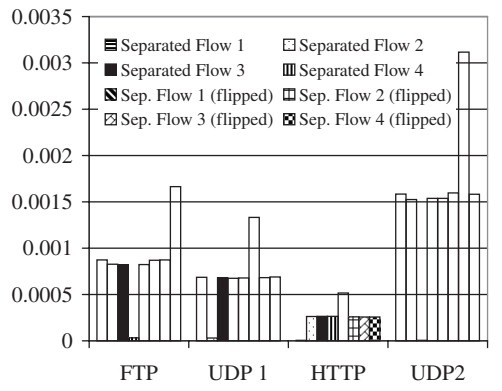

(b) MSE

Fig. 9. Performance of flow separation for different types of traffic (with multicast traffic).

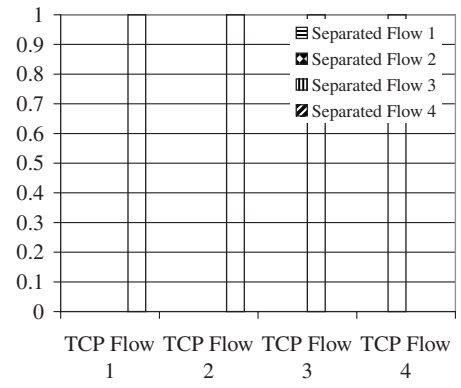

(a) Frequency spectrum matching degree

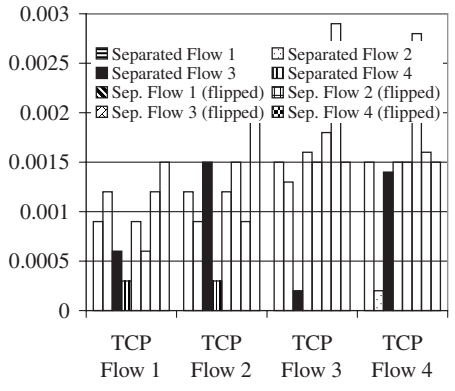

(b) MSE

Fig. 10. Performance of flow separation for TCP-only traffic (without multicast traffic).

two UDP flows have approximately similar periods, and the periodical behavior is not strong for exponential on/off traffic.

\subsection{TCP-Only Traffic}

Since most of the traffic in today's network is TCP, we focus on TCP traffic in the next series of experiments. All the flows in this experiment are FTP flows. To distinguish the flows, we vary the link delays between the sender and mix, with $S_{1}$ having $10 \mathrm{~ms}$ link delay to the mix, and $S_{2}$ having $15 \mathrm{~ms}$ delay. ${ }^{3}$

Figure 10 shows the flow separation performance. Since there is no multicast traffic, the redundancy in observations results that TCP Flow 1 and TCP Flow 2 are still mixed. In the final result, the flows are identified successfully by the frequency spectrum matching method.

\subsection{TCP-Only Traffic with Multicast Flow}

In this experiment, we change one FTP flow in the previous experiment to a multicast UDP flow. The UDP flow is exponential on/off traffic with the same parameters as UDP-1 of the experiment in Section 6.3.

${ }^{3}$ The difference in link delays gives rise to different round-trip times for the different TCP connections. As we show in the experiments in Section 8.2, BSS is just as effective in separating TCP flows when link delays are identical, and therefore, the round-trip times are very similar. 


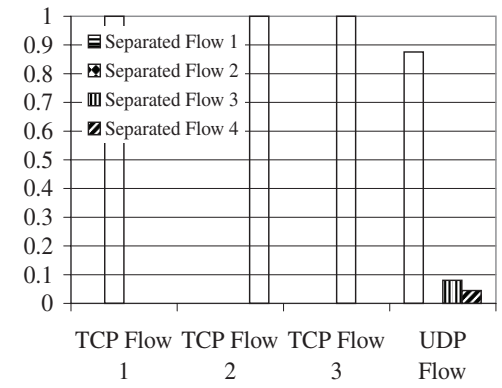

(a) Frequency spectrum matching degree

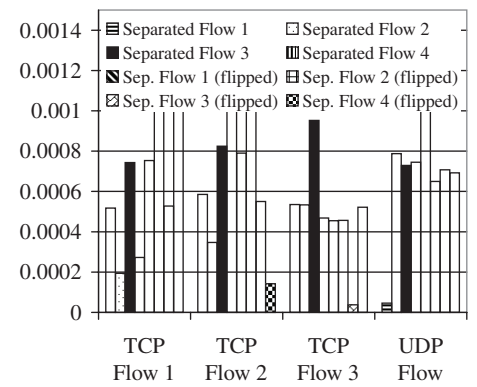

(b) MSE

Fig. 11. Performance of flow separation for TCP-only traffic (with multicast traffic).

Figure 11 shows the flow separation performance. Similar to the effect of multicast flows on different types of traffic, the four flows are now separated completely. We also observe that the frequency spectrum method identifies the FTP flows successfully. The performance for the exponential on/off UDP flow is not as good as for FTP flows, however, because the frequency signature of exponential traffic is very weak.

\section{EVALUATION OF SCALABILITY OF FLOW SEPARATION}

In order to gain insight into how flow separation performs as systems scale, we evaluate the flow separation performance with respect to (i) increasing numbers of flows in the mix-level aggregate flows (the number of aggregate flows remains constant), (ii) increasing numbers of mix-level aggregate flows, and (iii) increasing numbers of ports per mix. A detailed description of experiments with larger amounts of flows and larger mixes is given in our companion report [Zhu and Bettati 2007].

\section{EVALUATION OF FLOW SEPARATION ATTACK FOR MIX NETWORKS}

Flow separation can also be useful in mix networks with either local or partially global passive attackers. The attacker can perform flow separation between the available monitoring points, and then use the separation results to gather further information about the traffic. As an example of this approach, we show a set of experiments where the attacker correlates the separated aggregate flows to first derive the path taken by the flows and then to generate the traffic map of the flows in the network. We point out that the attacker can infer this information without having access to traffic information about any individual flow.

\subsection{Experiment Set-up}

Figure 12 shows the network set-up in this experiment. A total of eight FTP flows from senders on the left side are traversing the mix network. To distinguish these eight FTP flows, we incrementally add a $5 \mathrm{~ms}$ delay to links connected to each sender. In Section 8.2, we illustrate how we also get excellent 


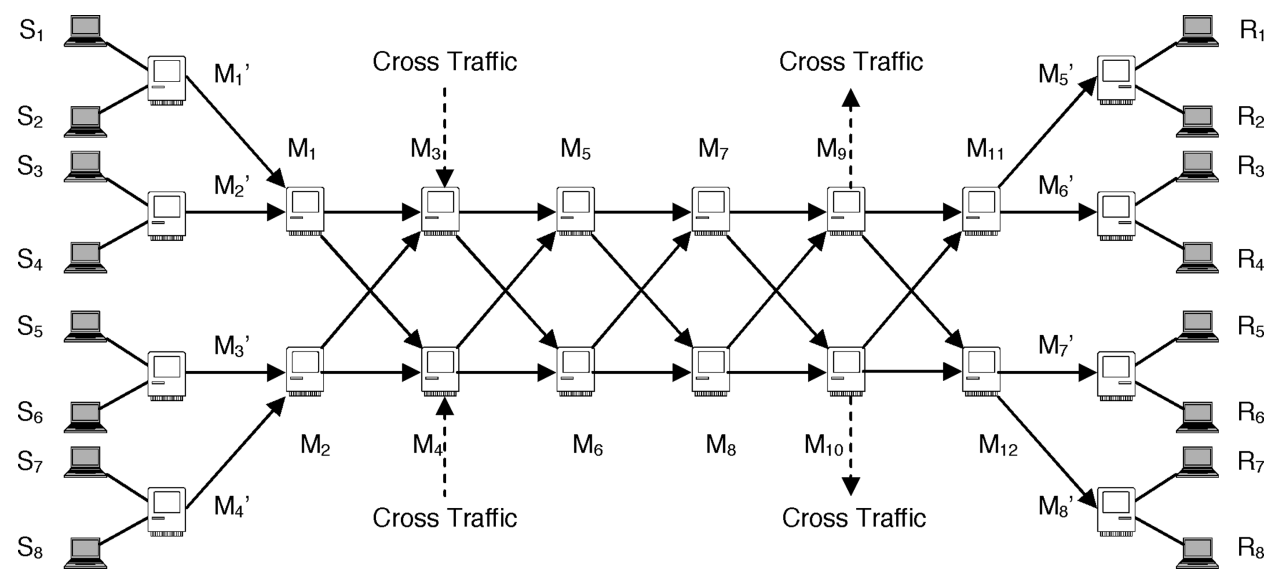

Fig. 12. Experiment set-up of mix network.

results with identical delays on links, and describe why round-trip times on connections have little effect on separation performance. To simulate the cross traffic in the mix network, four larger aggregates of flows are added to the mix network. In order to reflect the self-similar nature of the network traffic, the high-volume cross traffic is Pareto distributed. The configuration of the flows is described in our companion report [Zhu and Bettati 2007].

In the center of the mix network, the traffic volume ratio between link-level aggregate traffic and each individual flow from senders is at least $7: 1$. We assume that the attacker can observe links connected to Mix $M_{1}, M_{2}, \ldots, M_{12}$. Thus, a flow originating from $S_{1}$ can take $2^{6}$ possible paths.

To measure the accuracy of the flow path reconstruction based on flow separation, we use an entropy-based metric as follows. Suppose we are interested in flow $F_{x}$. The attacker can suspect the flow $F_{x}$ taking a path $P_{i}$ with probability $p_{i}$ based on the information gathered from the flow separation in the mix network. Assuming there are $h$ possible paths that can be suspected as the path taken by Flow $F_{x}$, we define the flow path obfuscation degree $D$ as

$$
D=-\sum_{i=1}^{h} p_{i} \log _{2} p_{i} .
$$

Suppose a flow originated from $S_{1}$ in Figure 12 is suspected to use each of $2^{6}$ possible paths with equal probability, then the path obfuscation degree $D$ is 6 bit.

In Zhu and Bettati [2007], we describe how simple flow separation, followed by dynamic programming based on frequency matching is very effective at identifying the flows in the system. While the obfuscation degrees of all flows $F_{1}$ to $F_{8}$ in the experiment have a value of 6 bits before the attack (i.e., all possible paths are equally likely in the six mix stages), the obfuscation degree after the attack drops to zero bit for all flows except for flows $F_{5}$ and $F_{7}$, where the the obfuscation degree drops to 0.5 bits. 


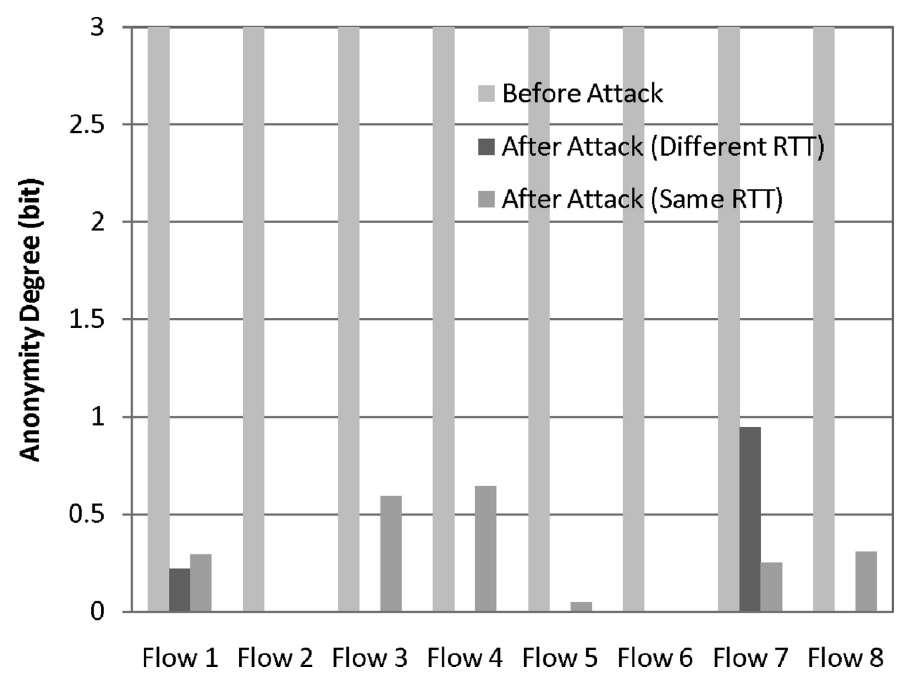

Fig. 13. Anonymity degree.

\subsection{Identical Round-Trip Time TCP Flows}

In this set of experiments, we illustrate that the level of variance in the roundtrip time does not affect performance of traffic flow separation. For example, an immediate (and, as it turns out, naïve) approach for a countermeasure would be to make the round trip times of traffic flows identical in order to increase dependence between traffic flows and, in turn, to render flow separation ineffective. In the following, we will show that flow separation remains effective for TCP flow even in the case of identical link delays (and, therefore, similar round-trip times). We reuse the experiment set-up used in the previous set of experiments but for a slight modification: The link delays of all links connected to senders are set to $5 \mathrm{~ms}$ instead of having the delays increase from link to link. As a result, the round trip times are very similar for all the eight traffic flows.

We measure the receiver anonymity by using the anonymity degree defined in [Serjantov and Danezis 2002] $]^{4}$ :

$$
D_{\text {rec }}=\sum_{i=1}^{n} p_{i} \log _{2}\left(p_{i}\right),
$$

where $p_{i}$ represents the probability that the $i$ th receiver is identified as the flow of interest, and $n$ is the number of possible receivers.

Figure 13 illustrates the performance of flow separation for the case of different RTTs and similar RTTs. We observe that separation performance for flows of similar RTTs and different RTTs is comparable. To investigate the cause for this comparable performance, we correlated the original traffic flows in both the identical-RTT experiments and the different-RTT experiments. (For a graphic

\footnotetext{
${ }^{4}$ Sender anonymity is defined similarly.
} 
of the results, see the companion report [Zhu and Bettati 2007].) During the first 10 seconds, the correlation across the traffic flows with similar RTTs is much higher than the correlation across traffic flows of different RTTs. After 10 seconds, the correlation among traffic flows of similar RTT is comparable with that of traffic flows of different RTTs. This decrease in dependence of traffic flows with similar RTTs explains the comparable performance in flow separation, as shown in Figure 13.

\section{EVALUATION OF PRIVACY ATTACKS ON WIRELESS NETWORKS}

Having illustrated the effectiveness of BSS to separate traffic flows in wired networks, we proceed to evaluate its effectiveness to separate, identify, and locate senders in anonymized wireless networks. Similar to the previous experiments, we perform a series of simulations in the ns- 2 network simulator.

\subsection{Experiment Set-up}

In the following experiments, we simulate a field with a $1,600 \mathrm{~m} \times 1,600 \mathrm{~m}$ square area. We assume that the sensors are arranged in a grid, and the distance between two neighboring sensors in the sensor grid is $50 \mathrm{~m}$. To eliminate boundary effects, the location of the wireless node is restricted to a $1,000 \mathrm{~m} \times$ $1,000 \mathrm{~m}$ center area of the simulated field. The wireless network interfaces of both wireless nodes and sensors are modeled according to the commercial Lucent WaveLan radio interface, which has a nominal radio range of $250 \mathrm{~m}$. For the sensors, the transmission function is disabled, so that they can only eavesdrop on the traffic. All simulations have a duration of 200 seconds. The packet count data is sampled with a sample interval of $50 \mathrm{~ms}$. We place 20 wireless nodes inside the center area. There are 36 randomly generated TCP flows in the wireless ad hoc network. To make sure that every wireless node sends packets, every wireless node has at least one TCP flow that originates from it. The size of the mini area is $50 \mathrm{~m} \times 50 \mathrm{~m}$, so that each area contains one sensor, and each sensor block is a $3 \times 3$ array of sensors.

\subsection{Effectiveness of Location Estimation}

Performance Metrics. As described in Section 5.3.2, the output of the location estimation algorithm is the suspected area of location of a node. To evaluate the performance according to the suspected area, we quantize the whole area using $5 \mathrm{~m} \times 5 \mathrm{~m}$ tiles. The suspected area is represented by a set of points inside the suspected area, each point representing the corner of the corresponding tile. Two metrics are used to evaluate the area. One is the mean error distance between the points inside the suspected area and the actual location of a wireless node. The other is the standard deviation of the error distance between the points inside the suspected area and the actual location of a wireless node. The first one measure the accuracy of the detection algorithm and the second measures the precision of the detection algorithm. If we cast the evaluation of the estimation algorithm in terms of evaluating a statistical estimator, the accuracy corresponds to the bias of the estimator and the precision corresponds to the variance of the estimator. 


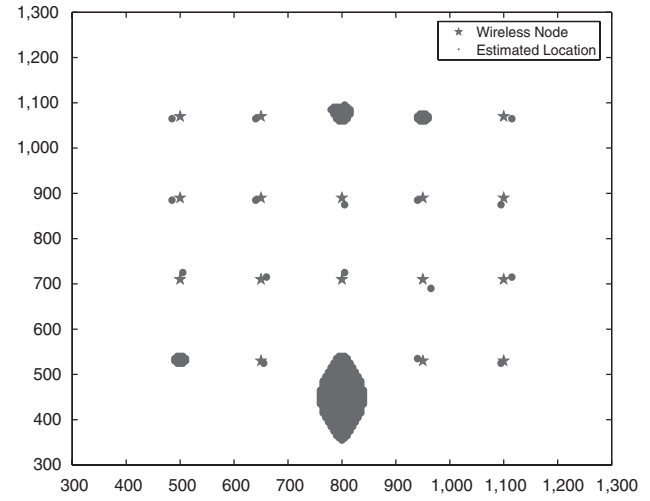

(a) Grid topology

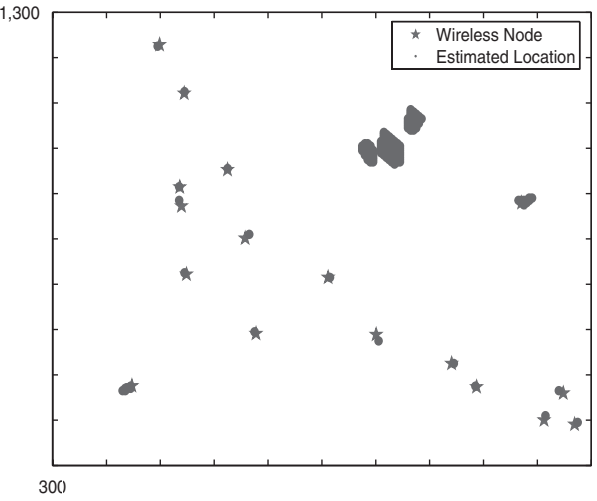

(b) Random topology

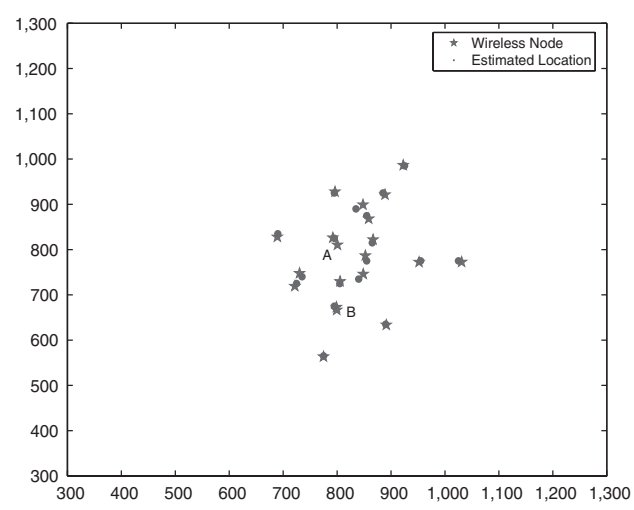

(c) Clustered topology

Fig. 14. Location estimation on different topologies. (Note: Two larger suspected areas are removed from (c) to prevent overlapping.)

To evaluate the intermediate result of the clustering step in Section 5.3.2, we compare the $K$ selected center components with the actual packet count time series of corresponding wireless nodes. The metrics used for comparison is the absolute value of the cross-correlation. We use absolute value here to account for the possibility that the separated component is of different sign than the time series.

Performance. We run our algorithm on three different types of node arrangements: grid, random, and clustered. Examples of typical results of our location algorithm are shown in Figure 14. Please note that in Figure 14(c), two relatively large suspected areas are removed to prevent overlapping with other suspected areas. The two larger suspected areas are caused by the two pairs of closely located nodes near Point A and Point B, respectively, in Figure 14(c). These closely located nodes cannot be differentiated by the sensor grid, so the number of actual differentiable nodes is less than the number of nodes we know.

To quantitatively evaluate our algorithm, we run simulations for both random and clustered arrangements. For clustered arrangements, the locations of 


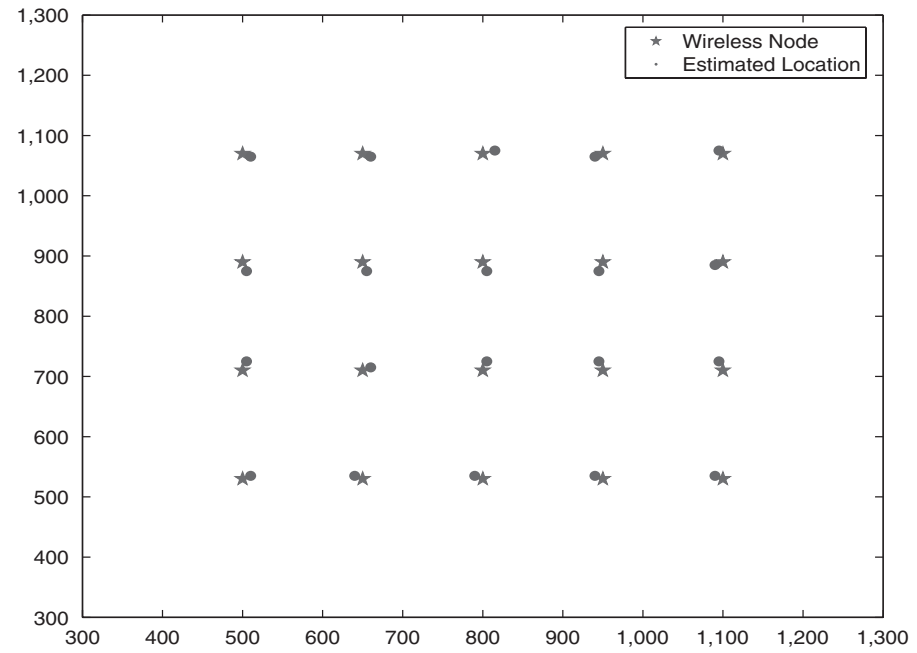

Fig. 15. Location estimation result (constant-rate traffic).

wireless nodes are generated by a Gaussian distribution with standard deviation of $100 \mathrm{~m}$ for both $x$ and $y$ axes. The mean of the distribution is chosen to arrange the wireless nodes around the center of the field. The results for clustered arrangements indicate that sparse arrangement of sensor grids yield good results as well. In Figure 14(c), for example, only about 300 sensors are needed to localize the nodes. (For a detailed description of accuracy and precision results from our experiments, please refer to our companion report [Zhu and Bettati 2007].)

Constant-Rate Traffic. In this experiment we evaluate our location detection algorithm against a network with constant-rate link padding. In such a network, packets are sent at constant intervals, and dummy packets are added whenever the link would idle. In the experiment, each wireless node sends constant-rate UDP packets to one of its neighbors. We use the grid topology of Figure 14(a). The choice of neighbor is made so that two loops are formed, with the outside nodes forming an outer loop and the inner nodes an inner loop. The packet sending rate of each wireless node is 40 packet/s and the bandwidth utilization is about $80 \%$. The goal of this set-up is to evaluate an arrangement that is as uniform as possible, thus making the separation and location problem maximally hard.

The results of this experiment are shown in Figure 15. We observe that the location detection algorithm is also effective against constant-rate traffic and heavy traffic. While the flows are constant-rate at sender application level, they are perturbed by the 802.11 MAC protocol, which adds enough timing signature to the flows and helps to separate the traffic. This experiment also illustrates that traffic padding at network layer or above is largely ineffective. A MAClevel traffic padding scheme that considers both the media control protocol and bandwidth efficiency is needed. 


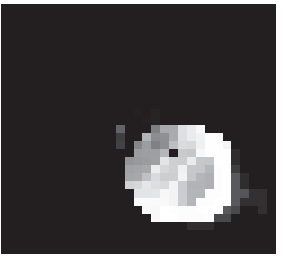

(a) Intensity image $I M G_{k}$

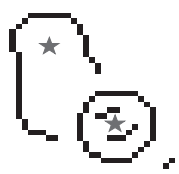

(b) Edge detection

Fig. 16. Sender/receiver/route anonymity attack.

\section{DISCUSSION}

\subsection{Countermeasures to Flow Separation Attack}

The countermeasures to flow separation attack are intuitive. First, padding of the links renders the observations obtained by the passive attacker nondistinguishable, or at least mostly redundant. Similarly, the use of pool-mix batching strategies would help. Pool mixes delay packets according to some probability distribution. If the delay probabilities are sufficiently small, the aggregate flows at the output ports can differ significantly from the aggregate flows at the input ports. This adds noise to the passive attacker's observations and can degrade the performance of flow separation attacks. The cost of such an approach is increased packet transfer latency and lower throughput, especially for TCP traffic. Next, the increase of the dependency among flows by adding dependent dummy traffic flows to the mix-level aggregate flows would reduce the effectiveness of BSS. Finally, the padding of aggregate flows to render the distribution of the packet counts Gaussian would render the BSS algorithms ineffective as well. Most BSS algorithms fail when the signals mixed are Gaussian distributed. Some classes of BSS algorithm, however, make use of the time structure of the signals, and can still separate the flows [Tong et al. 1991; Molgedey and Schuster 1994]. In general, BSS algorithms coping with noisy delayed signals and overcomplete base problems are still active research topics in BSS research. Flow separation attacks will be more powerful when more advanced such algorithms become available.

\subsection{Further Attacks on Wireless Networks}

The mechanisms used to estimate density and location information can be used to infer additional information about the wireless nodes as well.

Traditional sender/receiver/route anonymity. For a given intensity image $I M G_{k}$, we can apply an edge detection algorithm to reveal the sender/receiver relationship as well as information about the communication path. The result of an example attack is shown in Figure 16. From the intensity image shown in Figure 16(a), we can observe the relationship between the sender and the receiver. A contour of the route taken by a flow is shown in Figure 16(b). (The locations of the sender and receiver are marked with a star.)

Identity privacy. If a priori information is available about a wireless user, such as a model for communication or motion, the identity can be 


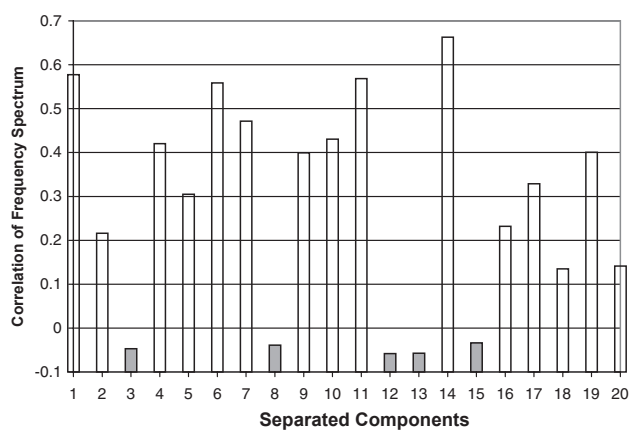

(a) Spectrum correction

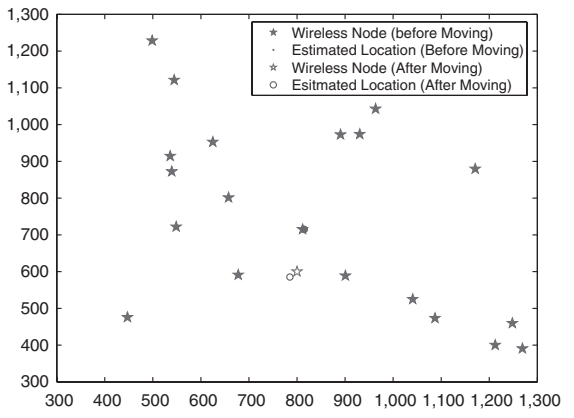

(b) Motion estimation

Fig. 17. Motion privacy detection.

derived by correlating the $K$ separated center components with the available models.

Motion privacy. To detect the motion of the wireless nodes in a field, we periodically compare the selected components by correlating them in the time domain and by tracking their estimated locations. When routes change and time correlation disappears, one can make use of spatial correlation until routes stabilize again.

Suppose the attacker finds out that component $R_{i}^{t}$ at some time $t$ is highly correlated to component $R_{j}^{t+\delta}$ at some later time $t+\delta$, and the location of component $R_{i}^{t}$ and $R_{j}^{t+\delta}$ is estimated to be $\operatorname{crea}_{i}^{t}$ and $\operatorname{crea}_{j}^{t+\delta}$, respectively. The attacker can infer that a node has moved from $\operatorname{crea}_{i}^{t}$ to $\operatorname{crea}_{j}^{t+\delta}$. From the analysis of node location privacy, we can get the location of $k$ nodes at time $t$ and $t+\delta$. Under the assumption that routing does not change dramatically from time $t$ to $t+\delta$, we can find the correspondence between $k$ signals at time $t$ and $k$ signals at time $t+\delta$ through correlation. When routes do change from time $t$ to time $t+\delta$, the timing behavior of flows can change as well, due to new contention situations or different path lengths. If routing does change, we can take advantage of correlation in the space domain. Since the speed of nodes is limited, for small values of $\delta$, the location of a node can not vary indeterminately.

Figure 17 shows the result of an experiment for the simple case of a single moving node. Figure 17(a) shows the correlation between the spectrum of the center component corresponding to the moving node before the move and the spectrum of all the center components after the move. The correlation is maximum between the spectrum of the center component corresponding to the moving node before the move and the spectrum of the center component (Component 14) corresponding to the moving node after the move. Figure 17(b) shows the motion estimation based on the two center components with maximum correlation.

\section{CONCLUSION}

In this article, we proposed anonymity attacks to both wired and wireless anonymity networks. These attacks are based on the BSS algorithms widely 
used to recover individual signals from mixtures of signals. Since the philosophy behind the design of current anonymity networks is to mix traffic or to hide in crowds, the proposed anonymity attacks are very effective.

We show in a series of experiments that flow correlation is effective in separating flows about which no a priori information is available. When several observation points are available throughout a network, we show that separation recreates the flow information at sufficiently accurate level so that frequencyspectrum correlation across observation points can reconstruct the path of the (unknown) flows. In the same way, we show how flow separation can also be used to simply recover the traffic map of the anonymity network. We discuss the possible usage of flow separation in different flow confidentiality settings, such as ssh tunnels or anonymity network settings, and we elaborate on criteria for its countermeasures.

Users of wireless networks are particularly exposed to privacy attacks (i.e., attacks that either identify the user or his presence or that identify the location of the user) since the communication medium is readily available for passive tapping. We show that traditional schemes for anonymous communication in wireless settings, such as masking of MAC addresses and link padding with dummy traffic, are largely ineffective against statistical timing analysis of network traffic. For example, we show how BSS allows us to identify the flows from different senders based on the same traces of aggregate packet counts. Similarly, PCA of traces of aggregate packet counts leads to accurate estimations of the number of nodes in a dense setting. We show in our experiments that the location estimation of nodes is accurate (typically significantly below the distance between any two sensors). These results indicate how effective these attacks are in separating traffic from different senders and identifying the presence of the senders.

The fact that the proposed schemes require from the sensors only the capability to receive and count 802.11 packets indicates that one should be able to deploy similar schemes for intrusion detection in ad hoc networks, for example: The ad hoc nodes could easily collect the data necessary to identify active intruders and to pin-point their location.

The poor performance of link-padding based anonymity protocols in wireless settings is due to a large part to the underlying carrier-sensing-based MAC protocols, which perturbs the originally padded traffic, and so renders it susceptible to BSS attacks. With privacy of users in mind, it may be time to re-evaluate the use of carrier-sensing-based versus scheduling-based MAC protocols and how to trade-off privacy versus efficiency in such systems.

\section{REFERENCES}

Bahl, P. and Padmanabhan, V. N. 2000. Radar: An in-building-based user location and tracking system. In Proceedings of the 19th Annual Joint Conference of the IEEE Computer and Communications Societies (INFOCOM'O0). IEEE, Los Alamitos, CA, 775-784.

Belouchrani, A., Abed-Meraim, K., Cardoso, J.-F., and Moulines, E. 1997. A blind source separation technique using second order statistics. IEEE Trans. Signal Process. 45, 2, 434-444.

Berthold, O. and Langos, H. 2002. Dummy traffic against long term intersection attacks. In Proceedings of the Privacy Enhancing Technologies Workshop (PET'02). Springer, Berlin, 110-128.

Berthold, O., Pfitzmann, A., and Standtke, R. 2000. The disadvantages of free MiX routes 
and how to overcome them. In Proceedings of the Designing Privacy Enhancing Technologies: Workshop on Design Issues in Anonymity and Unobservability. Springer, Berlin, 30-45.

CAI, J., You, H., Lu, B., Pooch, U., And Mi, L. 2005. Whisper: A lightweight anonymous communication mechanism in wireless ad-hoc networks. In Proceedings of Internation Conference on Wireless Networks (ICWN'05). 482-488.

CARdoso, J.-F. 1998. Blind signal separation: Statistical principles. In Proceedings of the IEEE $86,10,2009-2025$.

Cruces, S. And Cichocki, A. 2003. Combining blind source extraction with joint approximate diagonalization: Thin algorithms for ICA. In Proceedings of the 4th Symposium on Independent Component Analysis and Blind Signal Separation. Academic Press, Orlando, FL, 463-468.

Cuellar, J. R., John B. Morris, J., Mulligan, D. K., Peterson, J., and Polk, J. M. $2004 . \quad$ RFC 3693: Geopriv requirements. http://www.ietf.org/rfc/rfc36-93.txt.

Danezis, G. 2004. The traffic analysis of continuous-time mixes. In Proceedings of the Privacy Enhancing Technologies Workshop (PET'04). Springer, Berlin, 35-50.

Danezis, G., Dingledine, R., And Mathewson, N. 2003. Maxminion: Design of a type iii anonymous remailer protocol. In Proceedings of the IEEE Symposium on Security and Privacy. IEEE, Los Alamitos, CA, 2-15.

Danezis, G. And Serjantov, A. 2004. Statistical disclosure or intersection attacks on anonymity systems. In Proceedings of the 6th Information Hiding Workshop (IH'04). Springer, Berlin, 293-308.

Diaz, C., Seys, S., Claessens, J., and Preneel, B. 2002. Towards measuring anonymity. In Proceedings of Privacy Enhancing Technologies Workshop (PET'02). Springer, Berlin, 54-68.

Dingledine, R., Mathewson, N., And Syverson, P. 2004. Tor: The second-generation onion router. In Proceedings of the 13th USENIX Security Symposium. USENIX, Berkley, CA, 303-320.

Dusi, M., Gringoli, F., and Salgarelli, L. 2008. A preliminary look at the privacy of tunnels. In Proceedings of 17th International Conference on Computer Communications and Networks (ICCN'08). IEEE, Los Alamitos, CA, 1-7.

Elnahrawy, E., Li, X., AND Martin, R. P. 2004. The limits of localization using signal strength: A comparative study. In Proceedings of the 1st IEEE Communications Society Conference on Sensor and ad Hoc Communications and Networks. IEEE, Los Alamitos, CA, 406-414.

Goldschlag, D., Reed, M., and Syverson, P. 1999. Onion routing for anonymous and private internet connections. Comm. ACM 42, 2, 39-41.

Gruteser, M. And Grunwald, D. 2003. Enhancing location privacy in wireless through disposable interface identifiers: A quantitative analysis. In Proceedings of the 1st ACM International Workshop on Wireless Mobile Applications and Services on WLAN Hotspots. ACM, New York, 46-55.

Gülcü, C. And Tsudik, G. 1996. Mixing e-mail with Babel. In Proceedings of the Network and Distributed Security Symposium (NDSS '96). IEEE, Los Alamitos, CA, 2-16.

Guvenc, I., Chaouki T., Abdallah, R. J., and Dedeoglu, O. 2003. Enhancements to based indoor tracking systems using Kalman filters. In Proceedings of the GSPx and International Signal Processing Conference.

He, Z., YAng, L., Liv, J., Lu, Z., HE, C., AND Shi, Y. 2000. Blind source separation using clusteringbased multivariate density estimation algorithm. IEEE Trans. Signal Process. 48, 2, 575-579.

Helsingius, J. 1996. Press release: Johan Helsingius closes his Internet remailer. http://www.penet.fi/press-english.html.

HowARD, J. D. 1998. An analysis of security incidents on the internet 1989-1995. Ph.D. thesis. Carnegie Mellon University, Pittsburgh, PA.

Huang, P., Feldmann, A., And Willinger, W. 2001. A non-intrusive, wavelet-based approach to detecting network performance problems. In Proceedings of the Internet Measurement Workshop. ACM, New York, 213-227.

Hyvarinen, A. AND INKI, M. 2002. Estimating over-complete independent component bases for image windows. J. Math. Imag. Vis. 17, 2, 139-152.

Hyvarinen, A. AND OJA, E. 1997. A fast fixed-point algorithm for independent component analysis. Neural Comput. 9, 7, 1483-1492.

Jutten, C. ANd Herault, J. 1991. Blind separation of sources, part 1: An adaptive algorithmbased on neuromimetic architecture. Signal Process. 24, 1, 1-10. 
Kesdogan, D., Egner, J., and Bueschkes, R. 1998. Stop-and-go MIXes: Providing probabilistic anonymity in an open system. In Proceedings of the Information Hiding Workshop (IH'98). Springer, Berlin, 83-98.

Kong, J., Gerla, M., and Hong, X. 2003. A new set of passive routing attacks in mobile ad hoc networks. In Proceedings of the IEEE Military Communications Conference (MILCOM'O3). IEEE, Los Alamitos, CA, 796-801.

KonG, J. AND HonG, X. 2003. ANODR: Anonymous on demand routing with untraceable routes for mobile ad-hoc networks. In Proceedings of the 4th ACM International Symposium on Mobile Ad-Hoc Networking and Computing (MOBIHOC-03). ACM, New York, 291-302.

Levine, B. N., Reiter, M. K., Wang, C., And Wright, M. K. 2004. Timing attacks in low-latency mix-based systems. In Proceedings of the 8th International Conference on Financial Cryptography (FC'04). Springer, Berlin, 251-265.

Molgedey, L. and Schuster, H. G. 1994. Separation of a mixture of independent signals using time delayed correlations. Phys. Rev. Lett. 72, 23, 3634-3637.

Niculescu, D. AND Nath, B. 2004. Vor base stations for indoor 802.11 positioning. In Proceedings of the 10th Annual International Conference on Mobile Computing and Networking. ACM, New York, 58-69.

PAREKH, S. 1996. Prospects for remailers-where is anonymity heading on the internet. http://www.rstmonday.dk/issues/issue2/remailers

Pham, D.-T., Garrat, P., and Jutten, C. 1992. Separation of a mixture of independent sources through a maximum likelihood approach. In Proceedings of the 6th European Signal Processing Conference (EUSIPCO'92). Elsevier, The Netherlands, 771-774.

Porras, P., Saidi, H., and Yegneswaran, V. 2007. A multi-perspective analysis of the storm (peacomm) worm. Tech. rep., SRI International, Computer Science Laboratory, Menlo Park, CA. http://www.cyber-ta.org/pubs/StormWorm/.

RAYMOND, J. 2001. Traffic analysis: Protocols, attacks, design issues and open problems. In Proceedings of the International Workshop on Design Issues in Anonymity and Unobservability. Springer-Verlag, Berlin, 10-29.

Reiter, M. K. And Rubin, A. D. 1998. Crowds: Anonymity for Web transactions. ACM Trans. Inf. Syst. Secur. 1, 1, 66-92.

Rennhard, M. and Plattner, B. 2002. Introducing morphmix: Peer-to-peer-based anonymous internet usage with collusion detection. In Proceedings of the ACM workshop on Privacy in the Electronic Society (WPES'02). ACM, New York, 91-102.

Serjantov, A. AND Danezis, G. 2002. Towards an information theoretic metric for anonymity. In Proceedings of the Privacy Enhancing Technologies Workshop (PET'02). Springer, Berlin, 41-53.

Serjantov, A., Dingledine, R., And Syverson, P. 2002. From a trickle to a flood: Active attacks on several mix types. In Proceedings of the Information Hiding Workshop (IH'02). Springer, Berlin, $36-52$.

Sherwood, R., Bhattacharjee, B., ANd Srinivasan, A. 2002. p5: A protocol for scalable anonymous communication. In Proceedings of the IEEE Symposium on Security and Privacy. IEEE, Los Alamitos, CA, 58-70.

Tong, L., wen Liu, R., Soon, V. C., AND Huang, Y.-F. 1991. Indeterminacy and identifiability of blind identification. IEEE Trans. Circuits Syst. 38, 5, 499-509.

von Rickenbach, P. AND WatTenhofer, R. 2004. Gathering correlated data in sensor networks. In Proceedings of the Joint Workshop on Foundations of Mobile Computing. ACM, New York, 60-66.

ZHANG, Y., LiU, W., AND LoU, W. 2005. Anonymous communications in mobile ad hoc networks. In Proceedings of the 24th Annual Joint Conference of the IEEE Computer and Communications Societies (INFOCOM'05). IEEE, Los Alamitos, CA, 1940-1951.

Zhu, B., Wan, Z., Kankanhaldi, M. S., Bao, F., And Deng, R. H. 2004. Anonymous secure routing in mobile ad-hoc networks. In Proceedings of the 29th Annual IEEE International Conference on Local Computer Networks (LCN'04). Los Alamitos, CA, 102-108.

Zhu, Y. AND BetTATI, R. 2007. Compromising confidentiality in wireless network using sensors with limited information. In Proceedings of the IEEE International Conference on Distributed Computing Systems (ICDCS'07). IEEE, Los Alamitos, CA.

Post-print standardized by MSL Academic Endeavors, the imprint of the Michael Schwartz Library at Cleveland State University, 